\title{
Miron Costin - cronista e poeta
}

Adriana Senatore: Miron Costin.

Cronista e poeta di un'epoca buia. Bari 2019, 298 p.

\section{Abstract}

\section{Miron Costin - Chronicler and Poet}

The volume is based on the analysis of the historiographic and literary work of Miron Costin, who held positions of primary importance in the military, administrative and political life of the Principality of Moldavia during the central decades of the seventeenth century. In particular, are examined the narrative structures of his chronicles, which often are inspired by the creations of contemporary and previous Polish historians. Furthermore, the contribution to the affirmation of the latinity of the Romanian people is clarified, when the question had not yet been put on the agenda. Moreover, the echoes of Greek and Latin classicism are identified in the verses of the poem $L a$ vita del mondo (The life of the world), steeped in motifs and modules of the overflowing spirit of the Baroque era.

Key words: Romanian literature, Polish literature, Moldovan chronicles, Miron Costin

Parole chiave: letteratura romena, letteratura polacca, cronache moldave, Miron Costin 
Adriana Senatore, che per oltre quaranta anni ha insegnato lingua e letteratura romena all'Università di Bari, negli ultimi tempi ha concentrato l'interesse scientifico sugli autori del Seicento e del Settecento, riservando una serie di saggi e un volume all'analisi dell'opera linguistica e letteraria di Ion Budai-Deleanu. Ha altresì curato, tradotto e pubblicato nel 2015 La Zingareide, o l'Accampamento degli zingari, un poema di oltre 8300 versi che in precedenza era stato reso soltanto in lingua ceca e in lingua francese, ma in una versione rimata e, quindi non sempre rispettosa dell'originale.

Su Miron Costin (1633-1691), cronista e poeta moldavo, aveva già pubblicato nella rivista "Philologica Jassyensia" un saggio nel quale identificava gli agganci con la cultura classica greco-latina del suo poemetto La vita del mondo, tradotto in appendice. Nel volume appena uscito (Adriana Senatore: Miron Costin. Cronista e poeta di un'epoca buia. Bari. Cacucci Editore 2019) ha esteso lo studio all'intera attività dello scrittore, posta in diretto contatto con la realtà storica, politica e sociale della Moldavia del Seicento, nella quale aveva per oltre un trentennio svolto un ruolo decisivo, avendo operato ai vertici dello Stato, come militare, diplomati$\mathrm{co}$, amministratore e politico di alto rango. Proprio il coinvolgimento diretto nelle lotte politiche sarà causa della sua tragica morte, voluta dal principe Constantin Cantemir, il padre del grande storico e poligrafo Dimitrie.

Un lungo stralcio del volume è dedicato proprio all'analisi del rapporto di odio e amore che traspare nelle pagine di Cantemir, in particolare nel libello celebrativo del padre. Un altro argomento toccato con una certa ampiezza è quello dell'atteggiamento di Costin verso il mondo cattolico, che talvolta ha indotto a ipotizzane la conversione alla fede romana. In effetti, Costin aveva frequentato i collegi della Compagnia di Gesù, dove aveva ricevuto una solida preparazione umanistica che si coglie con chiarezza al fondo della sua scrittura. Nell'analizzare le opere storiche di Costin, che si propongono lo scopo evidente di rivendicare le origini latine del popolo romeno, Adriana Senatore ha cercato di individuarne i tratti artistici essenziali, in primo luogo, la propensione del cronista a narrare le vicende della piccola e amata patria in maniera plastica e coinvolgente, come appare dai non pochi brani in cui la narrazione assume il carattere e l'andamento di brevi scene drammatiche. Naturalmente, gran parte del volume è riservata all'analisi del poema, condotta sulla falsariga del saggio già pubblicato, ma ampliata con osservazioni e riferimenti approfonditi ed estesi a tutto il mondo classico.

Analizzando, infine, tra gli scritti minori di Costin tre orazioni indirizzate, anche a nome dei propri figli, al principe Antonie Ruset e all'erede al trono, Adriana Senatore è riuscita a esprimere una parola definitiva su un problema che aveva suscitato dubbi e interpretazioni diverse tra gli studiosi. Nei tre discorsi encomiastici, scritti in latino con frequenti rinvii alla mitologia (ed era la prima volta che un letterato romeno, sia pure non nella lingua materna, sollevava il velo di un 
mondo, quello degli dèi, delle ninfe, degli eroi della classicità fino ad allora ignoto ai lettori dei Principati), si accenna a un dono offerto al sovrano in occasione delle festività natalizie del 1676, e cioè un labirinto di verzura in cui ninfe e deità avrebbero danzato in onore dell'illustre destinatario. Vasile Urechia, curatore della prima edizione delle opere di Costin, considerava quel labirinto un testo scritto di un genere non meglio identificabile, in ogni caso, un frutto della penna dell'autore; Petre Panaitescu, lo slavista e critico della letteratura romena che più di altri ha contribuito nel Novecento alla conoscenza dell'opera del cronista e poeta moldavo, intese alla lettera le parole del testo e stimò reali il giardino e il labirinto che i Costin avrebbero approntato e offerto al voivoda. A parere della studiosa è però nel giusto Dan Horia Mazilu che, sulla scia dell'ipotesi di Urechia, ritiene quel dono uno scritto letterario e specifica che la metafora del labirinto aveva trovato all'epoca larga diffusione nelle lettere europee, da Jan Amos Komenský a Pedro Calderón de la Barca. E però non a componimenti di tali autori, concettosi e di profonda ispirazione spirituale occorre riferirsi, ma a quelli - veri e propri virtuosismi e arzigogoli di lettere e parole - che nell'atmosfera ampollosa del Barocco esaltavano celebri e influenti personaggi dell'epoca, del tipo di quelli che Juan Caramuel y Lobkowicz escogitò e raccolse nel volume Primus calamus... apparso a Roma nel 1663. Due dei suoi scherzi poetici, un "Panegyrin Labyrinthica" e un "Labyrinthus Metricus", nei quali l'ornamentazione silvestre fa pensare ancor più al genere dell'omaggio al principe di Moldavia, sono riprodotti alle pagine 134 e 135 del volume.

Come è noto, la figura di Costin interessa, e non poco, la stessa storia della cultura polacca: per sfuggire all'ira di Abbās pascià, lancu Costin, il padre del cronista che era tra gli esponenti più in vista della fazione polacca in terra moldava, era stato costretto a trasferirsi con la famiglia nei confini della Rzeczpospolita, dove morrà in esilio. In ricompensa dei preziosi servigi resi alla corona, la Dieta di Varsavia deliberò nel 1638 di conferire a lui e ai figli maschi, Alexandru, Miron e Potomir, l'indigenato, ossia la piena cittadinanza, e un titolo nobiliare trasmissibile. Nel paese che l'aveva accolto con tanta generosità, Miron frequenta i corsi di studio al collegio gesuitico di Bar, non più sperduto villaggio in riva al fiume Rów, ma munito avamposto militare e florido agglomerato mercantile dopo che la regina Bona Sforza, sollecita alle esigenze dello sviluppo economico e della sicurezza, gli aveva concesso larghe esenzioni fiscali e l'aveva cinto di possenti fortificazioni, come farà più tardi a Bari che reggerà con il titolo di duchessa. E nel nome del piccolo centro, allora appannaggio della regina e oggi situato nei confini della Repubblica di Ucraina, Bona volle richiamare proprio quello della città pugliese, sua patria d'adozione.

Ritornato in terra moldava con il mutare della situazione politica, Miron Costin percorse tutti i gradini di un brillante cursus honorum che culminò con la nomina a gran logoteta, il più stretto collaboratore del principe. Ai gravosi impegni

181

Fabrica Litterarum Polono-Italica | 2020, nr 1 (2) 
diplomatici, militari e amministrativi, l'eminente statista affiancò per tutta la vita un'intensa attività letteraria che comprende anche due opere in lingua polacca, un compendio della storia moldava (Chronika ziem moldawskich y multanskich), dove affronta per la prima volta la questione delle origini e della formazione del popolo romeno, intendendo confutare con puntuta vis polemica una tesi calunniosa che li voleva discendenti dai galeotti deportati dall'Impero romano nella Dacia di nuova conquista. L'opera, che si configura come un'epistola indirizzata al commesso della corona di Varsavia Marek Matczyński e a lui recapitata per il tramite di Jan Gniński, uno dei protagonisti della storia polacca di quel secolo, intendeva non soltanto presentare alle più alte autorità di Varsavia il retaggio millenario delle genti vissute sul suolo dell'antica Dacia, ma anche sottolineare le capacità difensive e le potenzialità strategiche dei due Principati, quello di Moldavia e quello di Valacchia, auspicandone la liberazione dal giogo ottomano.

L'altra opera, un poema sulle vicende moldave pervenuto in due varianti ( $\mathrm{Hi}$ storya polskimi rytmami o Wołoskiey Ziemi i Multanskiey e Historya o Wołoskiey Ziemi), fu composta in un frangente particolare: nel 1663 il principe di Moldavia Gheorghe Duca fu obbligato, al pari di altri tributari del sultano, a partecipare all'assedio della capitale dell'Impero asburgico, ma la campagna bellica si concluse con una rovinosa disfatta dell'esercito turco sotto le mura di Vienna, dove il re di Polonia Jan Sobieski e le forze alleate della Lega Santa spezzarono per sempre lo slancio di conquista della Sublime Porta. Mentre tentava di ritornare in patria il principe moldavo fu catturato da una pattuglia polacca e incarcerato insieme con vari dignitari di corte. A Costin, tuttavia, arrise un destino diverso perché il sovrano di Polonia, che lo conosceva e stimava, gli offrì asilo nel casino di caccia di Daszów. Nella quiete di quell'angolo dei Carpazi Miron si dedicò alla redazione del poema che volle aprire con una dedica al munifico protettore.

Entrambi gli scritti polacchi, ma il discorso può estendersi anche ai testi storici in romeno e, in certa misura, al poema La vita del mondo, sono chiara testimonianza di una peculiare temperie culturale, in cui si stabilirono e fiorirono proficue relazioni politiche, religiose e letterarie tra la Moldavia e la Polonia e, per tale versante, il più ampio Occidente cattolico. Nell'aura della predominante mentalità barocca, gli intellettuali moldavi si accostarono ai valori del mondo classico e dell'Umanesimo, una parentesi breve, spezzata ben presto dalla ripresa dell'influenza bizantina e russa, ma ricca di copiosi risultati nel campo delle lettere e delle arti.

Le strofe del poema hanno assicurato a Costin un posto sia pure minore nella stessa storia della cultura letteraria polacca: già nel $1820 \mathrm{fu}$ incluso nel primo volume del dizionario dei poeti (Dykcyonarz poetów polskich) pubblicato da Michał Hieronim Juszyński, che ne definiva il genere "wiersz heroiczny", collocandolo nell'ampia fioritura barocca dei poemi eroico-cavallereschi, come quelli che, per esempio, Samuel Twardowski aveva dedicato ai grandi eventi bellici del suo tempo

182

Fabrica Litterarum Polono-Italica | 2020, nr 1 (2) 
(Wojna chocimska e Wojna domowa z Kazaki y Tatary), segnati tutti da reminiscenze tassiane o, meglio, tasso-kochanowskiane. Nel primo volume della monumentale storia della letteratura polacca curata da Michał Wiszniewski in pieno Ottocento ne viene data una semplice segnalazione bibliografica («Mirona Kostyna, Opisanie ziemie mołdawskiéi i multańskiéi»); nei primi decenni del Novecento altri due storici letterari, Aleksander Brückner e Gabriel Korbut non trascurarono nelle rispettive trattazioni di menzionarne la creazione epica, fornendo concise indicazioni sulla vita e l'opera dell'autore. I tre storici della letteratura non si espressero sul valore artistico del poema che di certo non superava il livello dei numerosi altri componimenti consimili del Seicento polacco, avvinto e caratterizzato dalle lusinghe della poesia epica.

La fatica dell'autrice del volume va dunque apprezzata non soltanto per il puntuale studio su una incisiva personalità delle lettere e della storiografia romene, ma anche per l'ampio spazio riservato all'analisi delle relazioni tra la realtà storica e culturale della Polonia e quella dei Principati in un'epoca di grandi rivolgimenti e mutamenti come fu il Seicento nell'area balcanico-danubiana.

\section{Bibliografia}

BiEŃkOWSKA Danuta (1977): Literatura rumuńska. W: Dzieje literatur europejskich. T 1. Warszawa.

Chowaniec Czesław (1931): Miron Costin en Pologne. Contributions à l'année 1684-1685.

In: Inchinarea lui Nicolae lorga cu prilejul împlinirii vârstei de 60 ani. Cluj.

Costin Miron (1998): Latopis Ziemi Mołdawskiej i inne utwory historyczne. Tłum. Ilona Czamańska. Poznań.

LUPI Gino (1968): Storia della letteratura romena. Firenze.

MIRSKA-LASOTA Halina (1975): Mały słownik pisarzy rumuńskich. Warszawa.

SENATORE Adriana (2017): Ion Budai-Deleanu e i prestiti slavi: dalle enunciazioni teoriche alla prassi poetica. "Critica del Testo", XX, nr 2.

\section{Abstrakt \\ Miron Costin - kronikarz i poeta}

Artykuł poświęcony jest analizie dzieła historiograficznego i literackiego Mirona Costina, który był znaczącą postacią w życiu wojskowym, administracyjnym i politycznym Księstwa Mołdawii w środkowych dekadach XVII wieku. W szczególności zbadane

183

Fabrica Litterarum Polono-Italica | 2020, nr 1 (2) 
zostały struktury narracyjne jego kronik, ich wkład w kształtowanie się narodu rumuńskiego. W twórczości Mirona Costina docierają do nas echa klasycyzmu greckiego i łacińskiego, nasycone motywami i objawieniami wybuchowego ducha epoki baroku.

Słowa kluczowe: literatura rumuńska, literatura polska, kroniki mołdawskie, Miron Costin 